\title{
The effect of warning and prior instruction on short-latency cerebral potentials produced by muscle afferents in man
}

\author{
SC GANDEVIA, B McKEON, D BURKE \\ From the Unit of Clinical Neurophysiology, Department of Neurology, The Prince Henry Hospital and \\ School of Medicine, University of New South Wales, Sydney, Australia
}

SUMMARY This study examined the effect of a warning and of prior instruction on the early components of the cerebral potential produced by stimulation of the left posterior tibial nerve at the ankle. Early components of this potential are dominated by the activity in muscle afferents from the small muscles of the foot. In $50 \%$ of presentations, the stimulus to the posterior tibial nerve was preceded by an auditory cue. In some sequences subjects were required to move toes on the left foot immediately the stimulus was detected. Although subjects responded more rapidly to shocks which were preceded by a warning there was no statistically significant effect of the warning on the amplitude or latency of the early components of the muscle afferent cerebral potential. Prior instruction to respond to the stimulus also failed to change the cerebral potentials. This study suggests that the ability to respond more rapidly to "warned" stimuli is due to events "upstream" of the sensorimotor cortex rather than to enhancement of the volley arriving at the cortex.

Instruction to perform a particular movement is associated with changes in the discharge of cells in the sensorimotor cortex ${ }^{1}$ and changes in the bias of short-latency spinal reflexes. ${ }^{2-5}$ Thus there is evidence that prior instruction may alter the inputoutput relation not only of spinal motoneurons but also of corticospinal neurons which project to them. In addition, longer latency reflex responses to stretch of muscles in the upper limb can be preset according to whether the subject is instructed to resist or relax prior to the perturbation..$^{26-9}$ Such reflexes may involve a transcortical path ${ }^{10-13}$ although this is debated. ${ }^{14-16}$ Warning about the presence of a stimulus can reduce reaction time and potentiate proprioceptive reflexes; 591718 this is mediated through central mechanisms other than activation of gamma motoneurons. ${ }^{5}$

Psychophysical studies have shown that afferents from muscle can provide sensations of movement

Address for reprint requests: Dr SC Gandevia, Unit of Clinical Neurophysiology, Department of Neurology, The Prince Henry Hospital, P.O. Box 233 Matraville, Sydney, Australia 2036.

Received 11 August 1982 and in revised form 29 November 1982 Accepted 9 December 1982 and position ${ }^{19} 20$ (for reviews see refs 21,22 ). Shortlatency cerebral potentials have been produced by muscle stretch, tendon percussion and electrical stimulation of muscle afferents in the lower limb of human subjects. ${ }^{23-25}$ These studies have also shown that, in normal subjects, the early components of the cerebral potential produced by percutaneous stimulation of the posterior tibial nerve at the ankle probably reflect activity exclusively in muscle afferents. There may be no contribution from cutaneous afferents, not only because of their lower peripheral conduction velocity, but because their central action can be gated out by muscle afferents. ${ }^{25}$

The purpose of the present study was to determine whether the cerebral potential produced by stimulation of muscle afferents in the lower limb is altered by prior instruction or by anticipation. If preparation for a motor act enhances the effectiveness of central transmission of impulses from a relevant group of muscle afferents then the size of the muscle afferent potential might be expected to increase. Certainly during detection of liminal cutaneous stimuli to the fingers cerebral blood flow to the "hand area" increases ${ }^{26}$ and a facilitatory convergence between cutaneous afferents from adjacent fingers occurs. ${ }^{27}$ 
Throughout this study the size of cerebral potentials produced by electrical stimulation of the posterior tibial nerve has been about half maximal; this corresponds to a muscle afferent volley in the peripheral nerve of about one tenth of its maximum. ${ }^{28}$ This important methodological point ensures that the experiment was conducted on the steep, rather than the flat, insensitive part of the input-output relation for the projection of muscle afferents to the cortex.

\section{Methods}

Data were obtained in 15 experiments performed on six healthy adult subjects. Apart from two of the authors who participated in the study the subjects were unaware of the hypotheses under examination. Subjects were seated comfortably in a chair with the neck supported on pillows. During experimental runs (see below) the subjects viewed an oscilloscope placed below eye level. Cerebral potentials were recorded to stimulation of the left posterior tibial nerve in 11 experiments and to stimulation of the digital nerves of the left hallux in a further four experiments. Stimuli to the posterior tibial nerve were delivered through subdermal needle electrodes overlying the nerve at the ankle. Similar electrodes were used for stimulation of the digital nerves of the hallux. The stimuli were square-wave pulses of $0.2 \mathrm{~ms}$ duration. Unless otherwise stated the level of stimulation was adjusted so that it produced a cerebral potential which was about half the size of that produced by maximal tolerable stimulation. This ensured that the size of the cerebral potential had not "saturated". ${ }^{28}$ For the posterior tibial nerve the required stimulus level was usually near that which produced a liminal twitch of the intrinsic muscles of the foot. Cerebral activity was recorded using stainless-steel needle electrodes inserted subdermally at the vertex, with a forehead reference. The cranial reference was chosen to reduce the contribution from extracranial and subcortical midline structures. ${ }^{2329-32}$ The activity was amplified (gain 100000 ) and filtered with bandwidth of either $1.6 \mathrm{~Hz}-1.6 \mathrm{kHz}$ or $8 \mathrm{~Hz}-800 \mathrm{~Hz}$ depending on recording conditions. To determine the appropriate stimulus level, the responses to different levels of stimulation were averaged over $100 \mathrm{~ms}$ using a fixed-programme averager with an artefact rejection facility. The sampling rate was $5 \mathrm{kHz}$. Either 256 or 512 repetitions were averaged at a stimulus rate of $2 \mathrm{~Hz}$. During the subsequent experiment, analysis of data was performed on-line by a microprocessor which also controlled the experimental sequence (see below). It had an artefact rejection facility and a sampling rate of $3.9 \mathrm{kHz}$. Latencies were measured with the aid of a cursor. In some experiments the afferent volley was monitored at the popliteal fossa with surface electrodes as described previously. ${ }^{28}$

In the initial eleven experiments the effect of warning (anticipation) on the cerebral potential produced by stimulation of the posterior tibial nerve was studied. In the first part of these experiments some stimuli to the posterior tibial nerve were preceded by a warning click delivered through headphones ("warning-stimulus-response") and some were not ("stimulus-response"). This is referred to as sequence $A$. The overall cycle period was varied unpredictably from 1.5 to $3.5 \mathrm{~s}$ and the order of stimuli with and without warning was randomised. The interval between the warning and the stimulus was one second, as used in other studies. $^{5}$ Subjects were instructed to relax the foot and to use the click as a signal that a shock would follow a second later. Subjects were asked to respond immediately they felt a shock with a small but rapid flexion movement of the toes. This response involved the muscles innervated by the stimulated nerve (including abductor hallucis). The latency of the response was recorded either with surface electrodes over the tendon and motor point of abductor hallucis or with a small accelerometer strapped to the hallux. Subjects were given visual feedback of their performance and practised the paradigm for several minutes prior to data collection. Data were collected during sequences of 4 minutes with rest intervals of 2-3 minutes between sequences. During the sequence the experimenter monitored the motor response of the subject on a second oscilloscope. This allowed the latency of voluntary electromyographic activity (EMG) in abductor hallucis to be determined. In two subjects with prominent $\mathrm{H}$ reflexes to posterior tibial nerve stimulation, the direct motor response ( $M$ wave), $\mathrm{H}$ reflex and voluntary activity were measured by rectification and integration of the EMG of abductor hallucis (time constant $0 \cdot 2 \mathrm{~s}$ ). In the first six experiments (in four subjects) 250 300 responses to posterior tibial nerve stimulation, with and without warning were recorded. The effect of warning was determined by comparison of the cerebral response produced when an auditory cue preceded the stimulus with that produced when there was no cue (sequence A). Control experiments showed that there was no discernible long-latency auditory potential which occurred between 1.0 and $1.1 \mathrm{~s}$ after the click and which would have been superimposed on the posterior tibial cerebral potential. In the second part of these six experiments the same procedure was repeated except that the subject relaxed and was not required to respond to the stimuli to the posterior tibial nerve (sequence B). The effect of prior instruction was determined by comparison of cerebral responses during the second part of the experiment in which subjects relaxed with those produced in the first in which subjects responded to the stimuli. Because of the lengthy experiments required to collect the data for both sequences, only sequence $A$ was used in the subsequent five experiments. In these five experiments more repetitions were averaged $(500-600)$ to define the cerebral potentials.

In a further series of experiments in four subjects the effects of these manoeuvres on a cutaneous cerebral potential (produced by stimulation of the digital nerves of the hallux through needle electrodes) were contrasted with those on a muscle afferent cerebral potential (produced by stimulation of the posterior tibial nerve, as above) on the same day. The effects of a warning stimulus were studied in three experiments. In one the effect of prior instruction was also studied. As in the previous experiments the levels of stimulation were adjusted so that the cerebral potentials were half their maximal size. In two subjects the cutaneous study preceded the muscle afferent study and in the other two the order was reversed. Measurements were made of the amplitudes of the first positive deflection (onset to P1) and of the first positive-negative deflection (P1-N1). 


\section{Results}

As a major purpose of this study was to determine whether warning about the imminent occurrence of a stimulus altered the short-latency cerebral potential produced by the stimulus it was important to know whether the warning resulted in potentiation of the subjects' motor response to the stimulus. In all subjects reaction times following stimuli to the posterior tibial nerve for which there was no warning were consistently longer than those for which there was a warning. The range of reaction times varied between subjects (160-200 ms for unwarned stimuli and 135-170 ms for warned stimuli), but there was an average reduction of about $20 \mathrm{~ms}$ when warning occurred (range: $15-40 \mathrm{~ms}$ ). Thus the subjects' ability to respond rapidly to the stimuli was improved by the warning. A further index of subjects' anticipation of the stimuli was that they responded prematurely (before the stimulus) on about $1 \%$ of presentations.

In two subjects it was also possible to examine changes in the excitability of spinal reflexes as the stimuli to the posterior tibial nerve at the ankle produced distinct $\mathrm{H}$ reflexes in abductor hallucis. There were no significant changes in the size of the $\mathrm{H}$ reflex when the stimulus was associated with a warning (fig 1 , above). There was also no correlation between the size of the $\mathrm{H}$ reflex and the latency of the voluntary response of abductor hallucis: large $H$ reflexes were often associated with long reaction times and vice versa (fig 1 , below).

The onset of cerebral activity after the posterior tibial nerve stimuli occurred at an average $34.2 \mathrm{~ms}$ (range: $32.0-36.7 \mathrm{~ms}$ ); the first positive deflection at $39.8 \mathrm{~ms}(37.7-41.3 \mathrm{~ms})$ and the succeeding negative deflection at $48 \cdot 8 \mathrm{~ms}(44 \cdot 8-50 \cdot 1 \mathrm{~ms})$. These values are similar to those previously reported from this laboratory. ${ }^{28}$ Evidence that the early components of this potential represent activity in rapidly conducting afferents from the intrinsic muscles of the foot has been presented above. ${ }^{24} 28$ Moreover four of the subjects who participated in these experiments had participated in previous studies in which the waveform and latency of the cerebral potential to stimulation of a purely motor fascicle of the posterior tibial nerve at the ankle was shown to be similar to that produced by surface stimulation. ${ }^{24} 25$ In 11 experiments on six subjects provision of a warning resulted in a reduction in the amplitude of the early components of the muscle afferent cerebral potential, compared with the amplitudes of the unwarned control potentials (sequence $A$; see Methods). The mean $( \pm$ SEM) changes were a $9 \%( \pm 6 \%)$ reduction for onset-P1 and a $12 \%( \pm 5 \%)$ reduction for P1-N1. The absolute changes ranged from -0.67 to
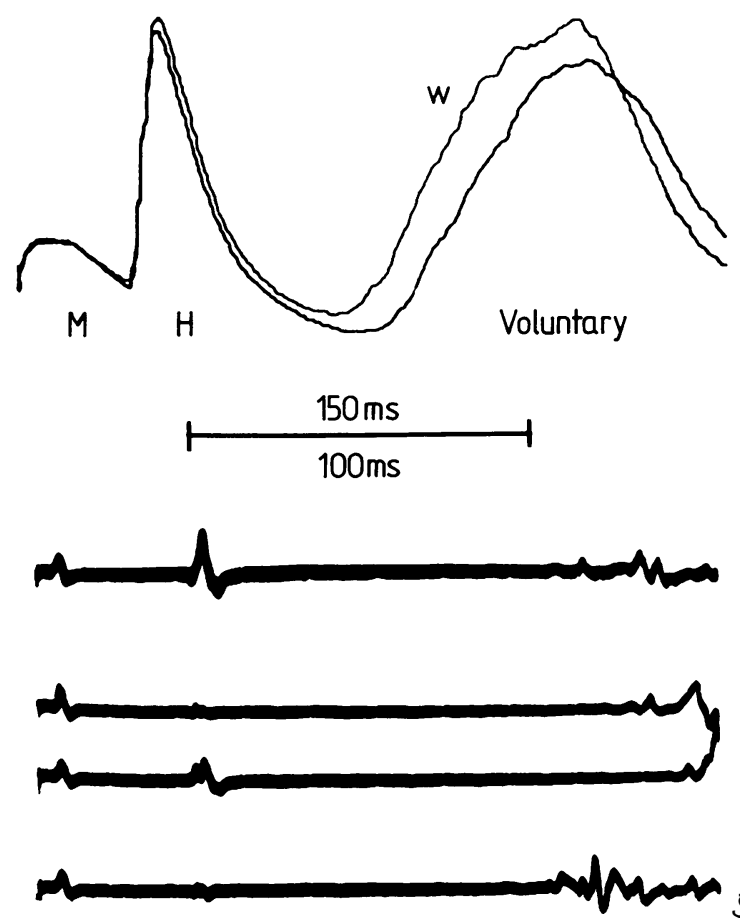

Fig 1 The upper trace shows the direct motor $(M)$, reflex $(H)$ and voluntary response of abductor hallucis to warned (w) and unwarned stimuli to the left posterior tibial nerve at the ankle. The recordings are average rectified and integrated EMG from the left abductor hallucis (50 trials). There was no difference in the amplitude of the direct motor or $H$ reflex response to the stimuli but the voluntary response appeared earlier in the warned than the unwarned trials. The lower traces show raw EMG from abductor hallucis during four consecutive trials which were preceded by a warning. The amplitude of the $\mathrm{H}$ reflex response does not correlate with the latency or size of voluntary activity. Calibration bar represents $150 \mathrm{~ms}$ in the upper trace and $100 \mathrm{~ms}$ in the lower trace. In this and subsequent figures $a$ negative potential is shown as an upward deflection.

$+0.51 \mathrm{uV}$ for onset-P1 and from -0.68 to +0.60 $\mathrm{uV}$ for P1-N1. Neither change was significant for the group of subjects (onset-P1: $0.2<p<0.3$; P1-N1: $0 \cdot 1<\mathrm{p}<0.2$; paired two-tailed Student's $t$ test). The size of the change varied between subjects and within any one subject when studied on different days. However it was comparable when repeated runs were performed in the same experimental session. In three of the 11 experiments there was a consistent reduction in onset-P1 amplitude of greater than $25 \%$ with warning and one in which the size increased with the warning. Results from a typical subject are shown in fig 2 . The latencies of the onset, $\mathrm{P} 1$ and N1 deflections were unaltered by warning. 


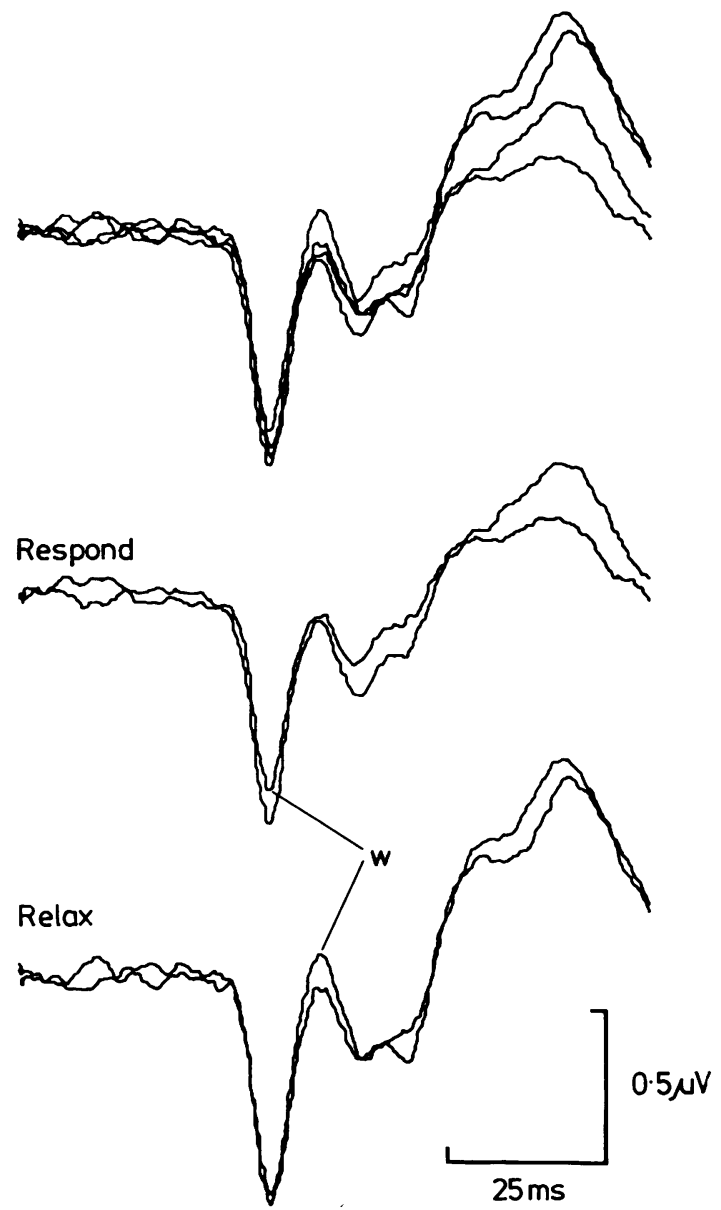

Fig 2 The upper trace shows the cerebral potentials recorded to stimulation of the left posterior tibial nerve during the four experimental conditions (that is sequences $A$ and $B$, see Methods). The middle and lower traces show the potentials recorded to stimuli during the "respond" and "relax" paradigms respectively. Traces marked "w" indicate that a warning preceded the stimuli to the posterior tibial nerve. There was little change in the latency or amplitude of the muscle afferent cerebral potential during the different experimental conditions. Each trace is the average of 500 responses.

To determine whether more obvious interactions occurred with very weak or maximal levels of stimulation, rather than the modest levels required to produce a cerebral potential of half-maximal size, experiments were performed across a range of stimulus intensities. Results from one such experiment are shown in fig 3 . Warning did not produce significant changes in amplitude of the cerebral potential at any stimulus level. In particular, with

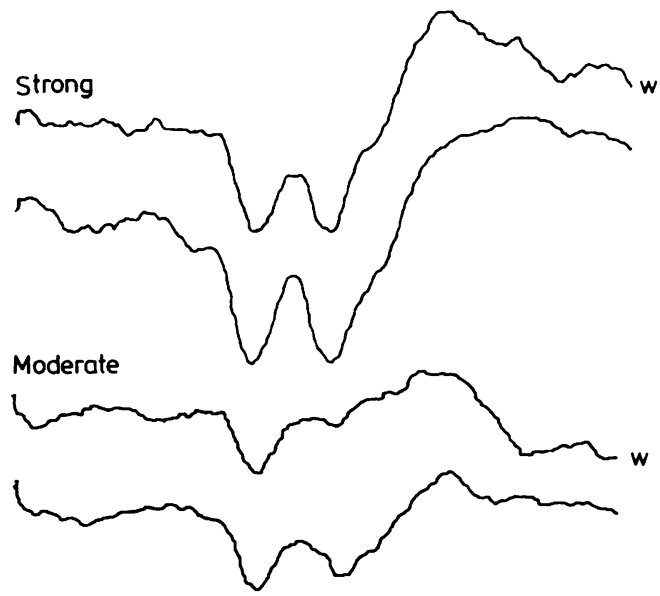

Weak

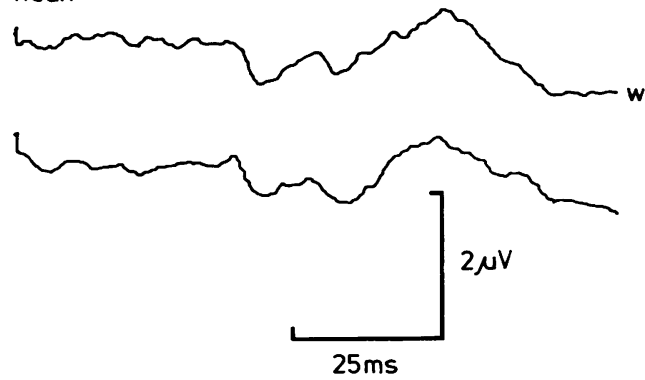

Fig 3 This shows the failure of warning to alter the latency or amplitude of the cerebral potential produced by stimulation of the posterior tibial nerve at three levels of intensity, weak, moderate and strong. It shows results from sequence A only (see Methods). Averages of 300 responses. Potentials preceded by a warning are labelled " $w$ ".

the weak stimulus the amplitude of the peripheral nerve volley was $10 \%$ that during maximal stimulation (fig 4), but there was no enhancement of the early components of the cerebral potential which might have been expected had transmission of the muscle afferent volley been enhanced by warning.

Similar findings emerged when the results of the first part of the experiment, in which the subject responded with a toe movement when he felt the posterior tibial nerve stimulus, were compared with those in the second part of the experiment in which he was instructed not to respond to the stimulus. Onset-P1 and P1-N1 amplitudes were not significantly or consistently altered by the prior instruction to respond (fig 2). Again, in the sequences in which the subject relaxed and did not respond to the stimuli, warning did not significantly alter the size of the control cerebral potentials 

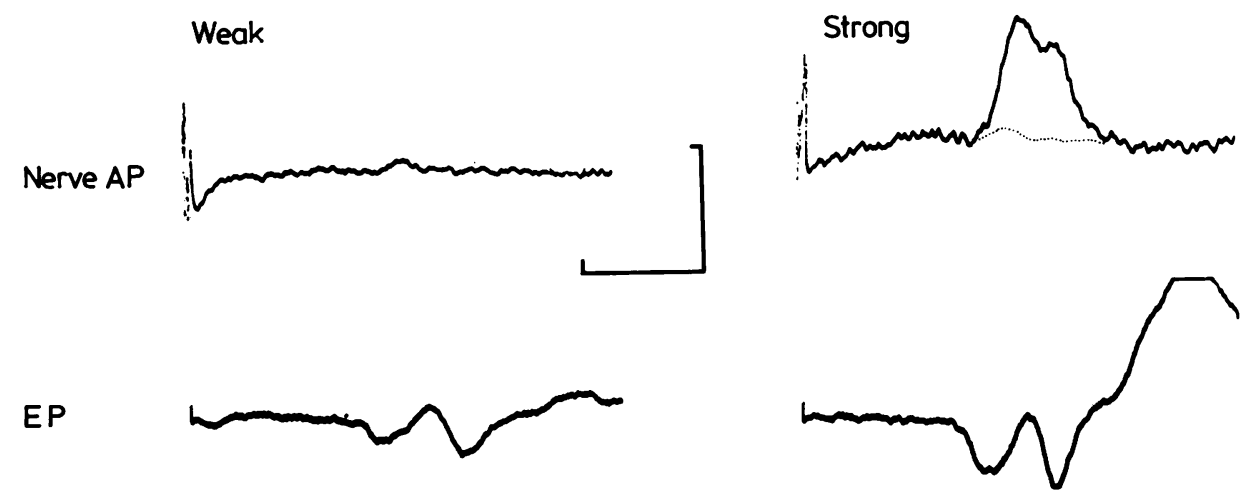

Fig 4 This shows the size of the afferent volley recorded at the popliteal fossa (above) and the cerebral potential it produced (below) for the weak and strong level of stimulation. There was a large increase in size of the afferent volley as the stimulus level was increased (including recruitment of a second peak) but the increase in size of the cerebral potential was relatively smaller. The peripheral nerve volley recorded to weak stimulation is dotted in at right. The results in this figure are from the subject in fig 3. Calibration: vertical, $2.5 \mu V$ for the nerve action potential and $4.0 \mu V$ for the cerebral potential; horizontal, $5 \mathrm{~ms}$ for the nerve potential and $25 \mathrm{~ms}$ for the cerebral potential.

(sequence B; see Methods).

To see whether a cutaneous potential was more modifiable than a muscle afferent potential, experiments were performed with stimuli to the digital nerves of the hallux and, separately, with stimuli to the posterior tibial nerve at the ankle in four subjects. There was no qualitative difference between the behaviour of the early components of the cutaneous and muscle cerebral potentials in the experimental sequences.

\section{Discussion}

The present study suggests that the overall input to the cerebral cortex from muscle afferents is not functionally enhanced by providing subjects with a warning that a stimulus is about to occur or by requiring subjects to respond with a movement when the stimulus is perceived. Only the early components of the cerebral potential produced by stimulation of the posterior tibial nerve were measured because these components (particularly onset-P1) are believed to represent activity associated with the projection of afferents to the cortical "leg area", ${ }^{23} 293032$ so that changes in these components are likely to represent modification of the afferent input at subcortical relay nuclei or on arrival at the cortex. Changes in longer latency potentials occur but they have not been of interest here as it is difficult to know what such changes represent. ${ }^{33} 34$ The posterior tibial nerve was used in this study because it has been established that stimulation of this mixed nerve produces a cerebral potential the early components of which are predominantly, if not exclu. sively, due to activity in muscle afferents from the small muscles of the foot. ${ }^{242528}$ There were no $a$ priori reasons to assume that the short-latency projection of muscle afferents to the cerebral cortex should behave in the same way as that for cutaneous afferents although this study shows that neither projection is greatly altered by warning or prior instruction.

It should be stressed that although there were no nett changes in the early components of the cerebral potential produced by stimulation of muscle or cutaneous afferents with warning or prior instruction, this does not imply that there were no changes in the relevant cortical neurons. ${ }^{1}$ It is possible that the technique of recording evoked potentials cannot resolve highly localised facilitatory activity (cf, however, ref 27) or that there was an equal balance of inhibition and excitation in the relevant neurons. Given these reservations, we assume, in subsequent discussion, that anticipation and prior instruction produce no overall change in the size of the afferent volley reaching cortex.

The consistent reduction in reaction time in the warned trials indicates that the subject was anticipating the imperative stimulus even if there was no enhancement of the muscle or cutaneous projection set up by the stimulus. This is consistent with the finding in the monkey that few primary somatosensory cortical cells display enhanced responsiveness during attentive behaviour. ${ }^{35}$ There was no correlation between the size of the $\mathrm{H}$ reflex of the responding muscle and the reaction time. It seems reasonable to conclude then that anticipation and prior instruction alter the motor programme at a local cerebral level "upstream" of the arrival of the affer- 
ent volley. The fact that changes in spinal reflex excitability can be dissociated from the reaction time suggests that attention (or anticipation) cannot be viewed as a unitary process, even under strict experimental conditions. ${ }^{36}$ If the muscle afferent cortical potential reflects the afferent limb of a transcortical reflex then it can be suggested that changes in the gain of the reflex as a result of "set" are not due to changes in afferent transmission.

As judged by changes in short-latency cerebral potentials many reports have demonstrated inhibitory or occlusive interactions between different afferent inputs 253738 or during active and passive movement..$^{38-41}$ Many of these findings could be due to the use of relatively strong stimuli as discussed elsewhere. ${ }^{28}$ However, as indicated above, the scalp-recorded cortical potential represents the average response of many cortical neurons rather than the response of a discrete group of neurons. As a result surrounding inhibition produced by one afferent volley could mask localised facilitatory activity produced by another.

This work was supported by the National Health and Medical Research Council of Australia. We are most grateful to Drs AK Lethlean and JW Lance for their support and comments on the manuscript.

\section{References}

${ }^{1}$ Tanji J, Evarts EV. Anticipatory activity of motor cortex neurons in relation to direction of an intended movement. J Neurophysiol 1976;39:1062-8.

${ }^{2}$ Hagbarth K-E. EMG studies of stretch reflexes in man. In: Widén L, ed. Recent Advances in Clinical Neurophysiology. Electroencephalogr Clin Neurophysiol 1967; Suppl 25:74-7.

${ }^{3}$ Evarts EV, Tanji J. Reflex and intended responses in motor cortex pyramidal tract neurons of monkey. $J$ Neurophysiol 1976;39:1069-89.

${ }^{4}$ Evarts EV, Granit R. Relations of reflexes and intended movements. In: Homma S, ed. Understanding the Stretch Reflex. Prog Brain Res 1976;44:1-14.

${ }^{5}$ Burke D, McKeon B, Skuse NF, Westerman RA. Anticipation and fusimotor activity in preparation for a voluntary contraction. J Physiol (Lond) 1980; 306:337-48.

${ }^{6}$ Hammond $\mathrm{PH}$. The influence of prior instruction to the subject on an apparently involuntary neuro-muscular response. J Physiol (Lond) 1956;132:17-18P.

${ }^{7}$ Lee RG. Tatton WG. Motor responses to sudden limb displacements in primates with specific CNS lesions and in human patients with motor system disorders. Can J Neurol Sci 1975;2:285-93.

${ }^{8}$ Colebatch JG, Gandevia SC, McCloskey DI, Potter EK. Subject instruction and the long latency reflex response to muscle stretch. J Physiol (Lond) 1979;292:527-34.
${ }^{9}$ Rothwell JC, Traub MM, Marsden CD. Influence of voluntary intent on the human long latency reflex. Nature 1980;288:496-8.

${ }^{10}$ Phillips CG. Motor apparatus of the baboon's hand. Proc Roy Soc Ser B 1969;173:141-74.

1 Evarts EV. Motor cortex reflexes associated with learned movement. Science 1973;179:501-3.

${ }^{12}$ Marsden CD, Merton PA, Morton HB. Is the human stretch reflex cortical rather than spinal? Lancet i:759-61.

${ }^{13}$ Choffon M, Lachat J-M, Rüegg DG. A transcortical loop demonstrated by stimulation of low-threshold muscle afferents in the awake monkey. $J$ Physiol (Lond) 1982;323:393-402.

${ }^{14}$ Ghez C, Shinoda Y. Spinal mechanisms of the functional stretch reflex. Exp Brain Res 1978;32:55-68.

15 Tracey DJ, Walmsley B, Brinkman J. "Long loop" reflexes can be obtained in spinal monkeys. Neurosci Lett 1980;18:59-65.

${ }^{16}$ Eklund G, Hagbarth K-E, Hägglund JV, Wallin EU. The "late" reflex response to muscle stretch: the "resonance hypothesis" versus the "long loop hypothesis". J Physiol (Lond) 1982;326:79-90.

${ }^{17}$ Brunia CHM, Vuister FM. Spinal reflexes as indicator of motor preparation in man. Physiol Psychol 1979; 7:377-80.

${ }^{18}$ Schier JGM, Brunia CHM. Effects of stimulus and task factors on Achilles tendon reflexes evoked early during a preparatory period. Physiol Behav 1982; 28:681-5.

${ }^{19}$ Goodwin GM, McCloskey DI, Matthews PBC. The contribution of muscle afferents to kinaesthesia shown by vibration-induced illusions of movement and by the effects of paralysing joint afferents. Brain 1972; 95:705-48.

${ }^{20}$ Eklund G. Position sense and state of contraction: the effects of vibration. J Neurol Neurosurg Psychiatry 1972;35:606-11.

${ }^{21}$ McCloskey DI. Kinesthetic sensibility. Physiol Rev 1978;58:763-820.

${ }^{22}$ Matthews PBC. Where does Sherrington's “muscular sense" originate? Ann Rev Neurosci 1982;5:189-218.

${ }^{23}$ Starr A, McKeon B, Skuse NF, Burke D. Cerebral potentials evoked by muscle stretch in man. Brain 1981;104:149-66.

${ }^{24}$ Burke D, Skuse NF, Lethlean AK. Cutaneous and muscle afferent components of the cerebral potential evoked by electrical stimulation of human peripheral nerves. Electroencephalogr Clin Neurophysiol 1981;51:579-88.

${ }^{25}$ Burke D, Gandevia SC, McKeon B, Skuse NF. Interactions between cutaneous and muscle afferent projections to cerebral cortex in man. Electroencephalogr Clin Neurophysiol 1982;53:349-60.

${ }^{26}$ Roland PE. Somatotopical tuning of postcentral gyrus during focal attention in man. A regional blood flow study. J Neurophysiol 1981;46:744-54.

${ }^{27}$ Gandevia SC, Burke D, McKeon B. Convergence in the somatosensory pathway between cutaneous afferents from the index and middle fingers in man. Exp Brain Res 1983; in press.

${ }^{28}$ Gandevia SC, Burke D, McKeon B. The relationship 
between the size of a muscle afferent volley and the cerebral potential it produces. J Neurol Neurosurg Psychiatry 1982;45:705-10.

${ }^{29}$ Tsumoto T, Hirose N, Nonaka S, Takahashi M. Analysis of somatosensory evoked potentials to lateral popliteal nerve stimulation in man. Electroencephalogr Clin Neurophysiol 1972;33:379-88.

${ }^{30}$ Jones SJ, Small DG. Spinal and sub-cortical evoked potentials following stimulation of the posterior tibial nerve in man. Electroencephalogr Clin Neurophysiol 1978;44:299-306.

${ }^{31}$ Eisen A, Elleker G. Sensory nerve stimulation and evoked potentials. Neurology (Minneap) 1980; 30:1097-105.

${ }^{32}$ Lueders H, Andrish J, Gurd A, Weiker G, Klem G. Origin of far-field subcortical potentials evoked by stimulation of the posterior tibial nerve. Electroencephalogr Clin Neurophysiol 1981;52:336-44.

${ }^{33}$ Lavine RA, Buchsbaum MS, Schechter G. Human somatosensory evoked responses: effects of attention and distraction on early components. Physiol Psychol 1980;8:405-8.

${ }^{34}$ Desmedt JE (ed). Cognitive components in cerebral event-related potentials and selective attention. Prog Clin Neurophysiol Basel: Karger 1979;6:322.

${ }^{35}$ Hyvarinen J, Poranen A, Jokinen Y. Influence of atten- tive behaviour on neuronal responses to vibration in primary somatosensory cortex of the monkey. $J$ Neurophysiol 1980;43:870-82.

${ }^{36}$ Bonnet M, Requin J. Long loop and spinal reflexes in man during preparation for intended directional hand movements. J Neurosci 1982;2:90-6.

${ }^{37}$ Abbruzzese $G$, Abbruzzese $M$, Favale $E$, Ivaldi $M$, Leandri M, Ratto S. The effects of hand muscle vibration on the somatosensory evoked potential in man: an interaction between lemniscal and spino-cerebellar inputs? J Neurol Neurosurg Psychiatry 1980;43:4337.

${ }^{38}$ Jones SJ. An "interference" approach to the study of somatosensory evoked potentials in man. Electroencephalogr Clin Neurophysiol 1981;52:517-30.

${ }^{39}$ Papakostopoulos D, Cooper R, Crowe HJ. Inhibition of cortical evoked potentials and sensation by self-paced movement in man. Nature 1975;285:321-4.

${ }^{40}$ Rushton DN, Rothwell JC, Craggs MD. Gating of somatosensory evoked potentials during different kinds of movement in man. Brain 1981;104:465-91.

${ }^{41}$ Abbruzzese G, Ratto S, Favale E, Abbruzzese M. Proprioceptive modulation of somatosensory evoked potentials during active and passive finger movements in man. J Neurol Neurosurg Psychiatry 1981; 44:942-9. 